\title{
Design and Performance of Broadband Dual Layer Circular Polarizer Based on Frequency Selective Surface for X-Band Applications
}

\author{
FARMAN ALI MANGI*, GHULAM ALI MALLAH**, SAEED AHMED KHAN***, AND DEEDAR ALI JAMRO* \\ RECEIVED ON 07.08.2015 ACCEPTED ON 22.11.2016
}

\begin{abstract}
A dual-layer circular polarizer based on FSS (Frequency Selective Surface) composed of two periodic strips in xoy plane. The transmission characteristic of present structure is comprehensively investigated that convert linearly polarized wave into circular polarized wave at operated frequencies. The designed structure has optimal performance for $x$-band applications. In addition, the designed model of single and dual-layer circular polarizer feature exceptionally strong circular polarization with low loss transmission and attracted for radar and satellite applications. The designed structures of circular polarizers are simple and can be easy fabricated as well. The transmitted waves are achieved with RHCP (Right Handed Circular Polarization) wave at $9.34 \mathrm{GHz}$ and LHCP (Left Handed Circular Polarization) wave at $10.73 \mathrm{GHZ}$. The corresponding axial ratio bandwidth is enhanced from 9.16$10.85 \mathrm{GHz}=16.89 \%$, respectively. Meanwhile, the proposed dual-layer circular polarizer achieves transmission with RHCP wave at $9.59 \mathrm{GHz}$ and axial ratio bandwidth is achieved from9.3-11.31 GHz = $19.49 \%$, respectively.
\end{abstract}

Key Words: Circular Polarizer, Dual-Layer Frequency Selective Surface, Fabry Perot Interferometer, Fractional Bandwidth, Quarter Wave Plate

\section{INTRODUCTION}

$\mathrm{P}$ olarization is one of the most important characteristics of microwaves that transfer the important information through one system to another system. Controlling of EM (Electromagnetics) waves propagation have been always desirable for the researchers to manipulate polarization state in transmission, reflection or absorption. Circular polarization has gained more attention in microwave communication and satellite communication. Circular polarization solves the great challenges, such as, atmospheric absorption, lower susceptibility and reflection effects as well. It is widely used in many electromagnetic waves applications, such as, optical communications and remote sensing.

In practical system, it is often realized to manipulate the polarization state of incident wave. For instance, circular polarizer can modify the polarization state to convert

\footnotetext{
* School of Physical Electronics, University of Electronic Science \& Technology, China.

** Department of Computer Science, Shah Abdul Latif University, Khairpur Mirs.

*** Department of Electrical Engineering, Sukkur Institute of Business Administration, Sukkur.
}

Mehran University Research Journal of Engineering \& Technology, Volume 36, No. 3, July, 2017 [p-ISSN: 0254-7821, e-ISSN: 2413-7219] 
linearly to circularly polarized wave. Moreover, it works as filter and polarization transformer which can pass the linearly incident wave into circular polarized wave. According to existing reported research contribution, circular polarization can be achieved by using various designed structures. In microwave technology, the design of circular polarizer based on meander line was introduced [1]. Various types of circular polarizer designs are composed of double-layer introduced by Euler [2] in which Fabry Perot interferometer approach has been employed to adjust the distance between the surfaces of layers [3]. According to existing research, circular polarizer can be designed by employing photonic [4-5], chiral structures [6-8], metasurface, metamaterials [9], grating structures [10,11], quarter wave plate [12], etc.

FSSs are introduced as circular polarizer, filter for many applications [13-16]. FSSs have good advantages of ease fabrication and low profile to make them more attractive among periodic structures. The classical approach was introduced to design FSS based polarizer which, is composed of cross layer design to use for circular polarization. Widening band-width is one of the important aspect, which has aroused the interest of researchers in the field of modern communication and various significant techniques have been employed to obtain broadband of polarizer.

The double-layer polarizer approach was introduced to obtain circular polarization with $3 \mathrm{~dB}$ axial ratio corresponding to $5.7 \%$ on operational bandwidth [17]. The single-layer Jerusalem cross FSS design was presented in which the $3.4 \%$ fractional bandwidth performance was achieved at frequency bands, corresponding $3 \mathrm{~dB}$ axial ratio and transmission loss 3.1 $\mathrm{dB}$ was observed at $17.8 \mathrm{GHz}$ [18].

Multi-band circular polarizer which is constructed of 2x2 strip array printed on both sides of the substrate. It achieved RHCP waves and LHCP waves at four distinct frequencies and accumulative calculated axial ratio was obtained of 8.16\% ranging from 8-12 GHz [19]. A broadband FSS dual-layer circular polarizer based on quarter wave plate demonstrated the axial ratio bandwidth of $14.11 \%$ over the frequency bands for circular polarization [20].

Recently, FSSs polarizers are presented with narrow bandwidth performance corresponding to modest transmission efficiency. Therefore, research is needed to minimize the transmission loss and improve the bandwidth widening of the polarizer to achieve the pure circular polarization. The open boundaries of air box/waveguide are applied along z-direction and size of air box along zaxis is selected as $68 \mathrm{~mm}$ in simulation models of singleand dual-layer circular polarizers. It is possible to obtain the corresponding all vector components of the electromagnetic wave by changing the size of unit cell along z-axis. The simulation results show that the structure can manipulate EM waves at lower or higher frequencies simply by changing the size of the unit cells [21]. Moreover, the resonant frequencies of the planar structure can be manipulated due to the geometry scalability [22].

In this work, we presented the design of single and duallayer circular polarizer structures based on FSS for Xband applications. The proposed dual-layer polarizer is based on two periodic strips which are printed on the both sides of two substrates which are oriented at different directions in xoy planes.

The simple efficient techniques are introduced to design structures to improve the bandwidth widening with low loss transmission at resonance frequencies. Comparing to some existing design of circular polarizers, our designed polarizer has significant advantages, such as, broadband widening, simple structure, easy fabrication and good circular polarization. Moreover, it is analyzed in detail that the transmission characteristics, and good axial ratio

Mehran University Research Journal of Engineering \& Technology, Volume 36, No. 3, July, 2017 [p-ISSN: 0254-7821, e-ISSN: 2413-7219] 
corresponding phase differences are achieved on operated frequencies.

\section{DESIGN PROCESSAND SIMULATION SETUP}

The designed structure of single and dual layer circular polarizer realize the linear-to-circular polarization convertors. Both simple low profile structures are designed in xoy plane in different directions. Fig. 1 depicts the unit cell of single-layer circular polarizer oriented at $45^{\circ}$ along xoy plane, respectively. The relative permittivity and thickness of the substrate are represented by $\varepsilon_{\mathrm{r}}$ and $\mathrm{h}$. The width of single strip is $\mathrm{w}$ and length is $\mathrm{l}$. where, the periods are $\mathrm{p}_{\mathrm{x}}$ and $\mathrm{p}_{\mathrm{y}}$ in $\mathrm{x}$ and $\mathrm{y}$ directions, respectively.

In Fig.1, single metallic strip is sticked on the substrate which is tilted at $45^{\circ}$ in xoy plane. In Fig. 2, two periodic metallic strips are printed on the top and bottom of the two individual dielectric substrates slanted at 45 and $90^{\circ}$ in xoy plane, respectively. The $\mathrm{r}$ parameter is represented the distance between two strips which are separated from each other at different directions in xoy plane as shown in Fig. 2. Therelative permittivity and thickness of the substrate are represented by $\varepsilon_{\mathrm{r}}$ and $\mathrm{h}$. The width of single strip is $\mathrm{w}$ and length is $\mathrm{l}$. where, the periods are $\mathrm{p}_{\mathrm{x}}$ and $\mathrm{p}_{\mathrm{y}}$ in $\mathrm{x}$ and $\mathrm{y}$ directions, respectively.

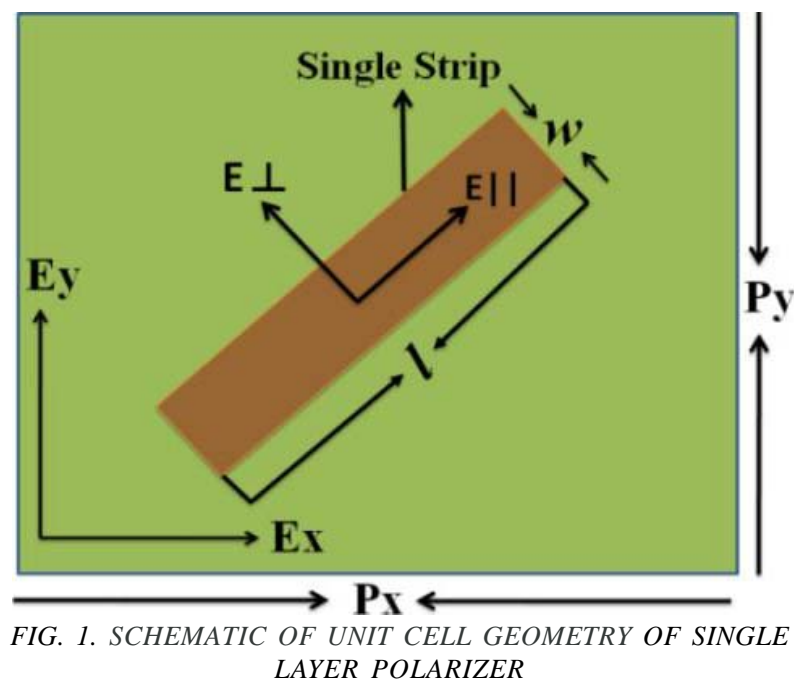

By using simulation, a broadband $\mathrm{x}$-linearly polarized wave impinges on the metallic strips as excitation source through floquet port- 1 along $+\mathrm{z}$ direction and assigns the unit cell boundary conditions of Master and Slave for designed model. The printed periodic strips on the substrate are assigned by PEC (Perfect Electric Conductor).The structure parameters of single and dual layer polarizers are presented as follows; $\mathrm{px}=25, \mathrm{py}=25, \mathrm{l}=11, \mathrm{w}=3.74 \mathrm{~mm}$, substrate Roger RT/duroid5880, $\varepsilon_{\mathrm{r}}=2.2$ and loss tangent of 0.0009 , $\mathrm{t}=0.508, \mathrm{r}=2 \mathrm{~mm}$, respectively as shown in Figs. 3-4.

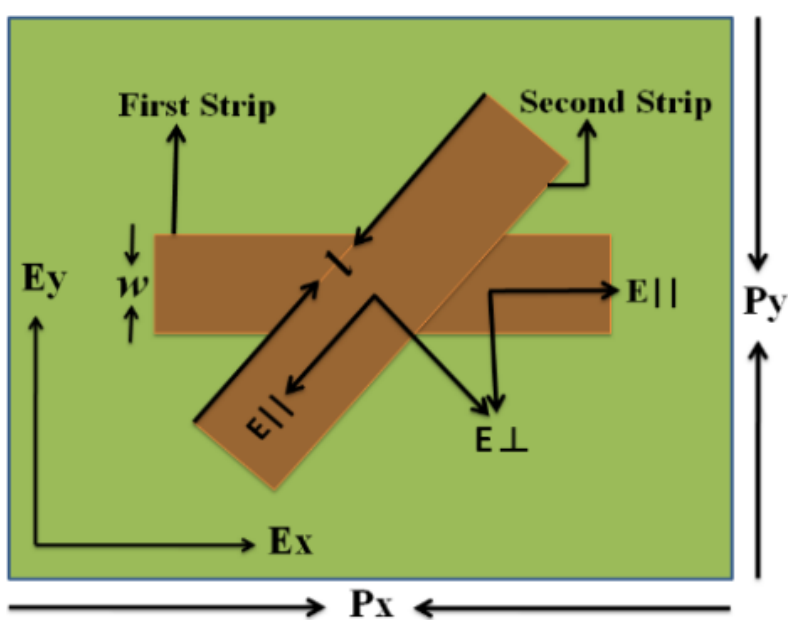

FIG. 2. SCHEMATIC UNIT CELL GEOMETRY OF DUAL LAYER POLARIZER

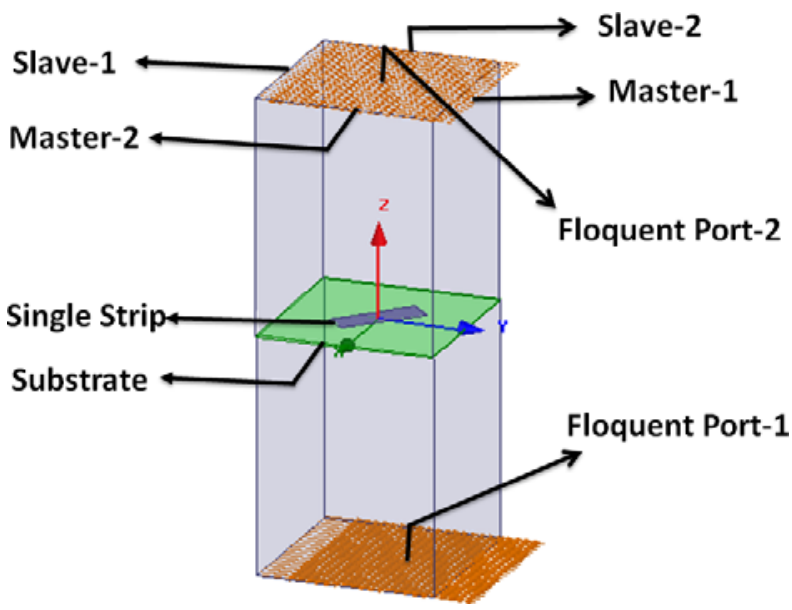

FIG. 3. A SAMPLE OF SINGLE-LAYER POLARIZER WHICH CONSISTS OF ONE PERIODIC STRIP PRINTED ON THE SUBSTRATE

Mehran University Research Journal of Engineering \& Technology, Volume 36, No. 3, July, 2017 [p-ISSN: 0254-7821, e-ISSN: 2413-7219] 


\section{RESULTS AND DISCUSSION}

It is assumed that the metallic strips are illuminated by linearly polarized wave Eialong $+\mathrm{z}$ direction and it decomposed into two orthogonal components $(\mathrm{E} \|$ and $\perp$ ) having equivalent magnitudes corresponding $90^{\circ}$ phase difference.

$$
\begin{aligned}
& E(t)=\hat{x} E_{X}(t)+\hat{y} E_{Y}(t)=\tilde{E}=\hat{x} \tilde{E}_{X}+\hat{y} \tilde{E}_{Y} \\
& \mathbf{E}_{\mathbf{x}}(\mathbf{t})=\operatorname{mx} \cos (\omega \mathrm{t}-\mathrm{Kz}) \\
& \mathbf{E}_{\mathbf{y}}(\mathbf{t})=\operatorname{my} \cos (\omega \mathrm{t}-\mathrm{Kz}+\delta)
\end{aligned}
$$

The polarization pattern is represented by the $\mathrm{x}$ and $\mathrm{y}$ subscript, $\mathrm{k}$ is the wave number, $\mathrm{t}$ is the time, $\delta$ is the phase offset between the two waves and $\omega$ is the frequency. The proposed structure generates the transmission with RHCP wave and LHCP wave.

The incident $\mathrm{x}$-linearly polarized wave through floquet port 1 along $+z$ direction produces resonance on the surface of bottom strip and decomposed into two orthogonal components. These two transmitted vector orthogonal components propagate with different velocities through

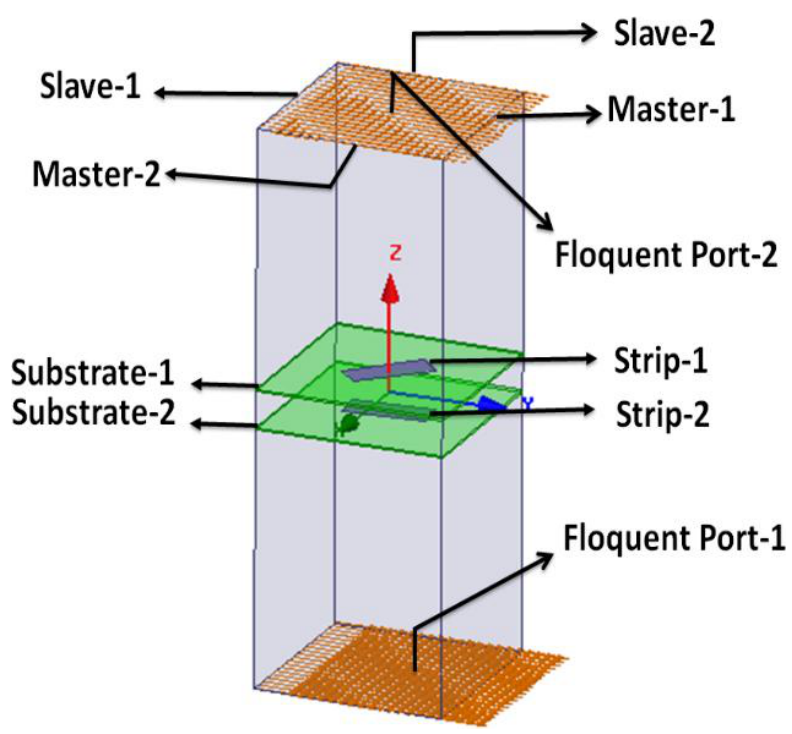

FIG. 4. A SAMPLE OF DUAL LAYER POLARIZER WHICH CONSISTS OF TWO PERIODICSTRIPS PRINTED ON BOTH SIDE OF INDIVIDUAL SUBSTRATE AND SEPARATED BY THE DISTANCE circular polarizer along $\mathrm{x}$ and ydirections such that the resultant wave is circular polarized wave at the other end of polarizer. Similarly, the transmitted polarized wave impinges on the top layer of strip to reflect LHCP wave and transmit one RHCP wave because of the low resonance produce on the top layer. Moreover, two transmitted frequencies can be generated with RHCP wave and LHCP wave at 9.34 and 10.73 GHZ, respectively.

Whereas, the clockwise rotation and anticlockwise rotation are determined to RHCP wave and LHCP wave when outgoing wave are transmitted from the output interface of the strip assuming the polarizer is illuminated by the incidence of $x$-linearly polarized wave with polarization angle $\theta=0^{\circ}$.

$\Delta \phi=\phi y-\phi x= \begin{cases}+\left(2 n+\frac{1}{2}\right) \pi & \text { for RHCP } \\ -\left(2 n+\frac{1}{2}\right) \pi & \text { for LHCP }\end{cases}$

The following equation denotes the equal magnitudes of RHCP wave and LHCP wave transmitted at the end of the polarizer.

$$
\begin{aligned}
& E_{0}(z, t)=\frac{E_{0}}{\sqrt{2}} \cos (K z-\omega t) \hat{x}+\frac{E_{0}}{\sqrt{2}} \sin (K z-\omega t) \hat{y} \\
& E_{0}(z, t)=\frac{E_{0}}{\sqrt{2}} \cos (K z-\omega t) \hat{x}-\frac{E_{0}}{\sqrt{2}} \sin (K z-\omega t) \hat{y}
\end{aligned}
$$

The fractional axial ratio of two orthogonal vector components is represented as:

$$
\begin{aligned}
& q=\frac{\left|E_{x}\right|}{\left|E_{y}\right|} \\
& \left|E_{x}\right| \cdot T^{x}=\left|E_{y}\right| \cdot T^{y}
\end{aligned}
$$

Whereas, the axial ratio is equal to 1 and $\mathrm{T}^{\mathrm{X}}$ and $\mathrm{T}^{\mathrm{Y}}$ indicate the amplitude of transmitted wave $\mathrm{E}_{\mathrm{X}}$ and $\mathrm{E}_{\mathrm{Y}}$. It means the circular polarization is achieved by the structure. 
$\left[\operatorname{ang}\left(E_{y}\right)+\operatorname{ang}\left(T^{y}\right)\right]-\left[\operatorname{ang}\left(E_{x}\right)+\operatorname{ang}\left(T^{x}\right)\right]= \pm n \pi / 2$

Where $n=1,2,3$,

On the other side of the structure, the transmitted E field decomposed in to two orthogonal components having equal magnitudes for circular polarization.

$\left|\mathrm{T}^{\mathrm{x}}\right|=\mathrm{T}^{\mathrm{y}} \mid$

$\left[\operatorname{ang}\left(T^{y}\right)-\left[\operatorname{ang}\left(T^{x}\right)\right]= \pm n \pi / 2\right.$

The reflection effects of the two layers of structure can be minimized by decreasing the length, width and distance between strips. The perfect fractional axial ratio of transmitted two components can be realized the quality of circular polarization from the other side of structure. In general, the axial ratio between $0-3 \mathrm{~dB}$ is acceptable in order to achieve circular polarization.
Fig. 5 depicts fractional axial ratio of the $E_{x}$ and $\mathrm{E}_{\mathrm{y}}$ components is achieved 1.5 at $9.3 \mathrm{GHz}$ and1.6 at 10.73 GHz. Meanwhile, the axial ratio bandwidth is obtained ranging from $9.16-10.85 \mathrm{GHz}=16.89 \%$ bandwidth.

In Fig. 6 the transmission loss of structure can be observed $-2.19 \mathrm{~dB}$ at $9.34 \mathrm{GHz}$ and -1.6 at $10.73 \mathrm{GHz}$ versus frequency bands, respectively.

When the circular polarizer is illuminated by the normal incident of x-linearly polarized wave, the outcome two linear orthogonal vector components transmitted from other side of circular polarizer having equal magnitudes corresponding $\pm 90^{\circ}$ phase difference over the frequency bands. It means that the transmitted waves are achieved with RHCP wave and LHCP wave. The phase difference of two transmitted linearly polarized waves is obtained $90.9^{\circ}$ at the $9.34 \mathrm{GHz}$ and $-90.4^{\circ}$ at $10.73 \mathrm{GHz}$ as represented in Fig. 7.

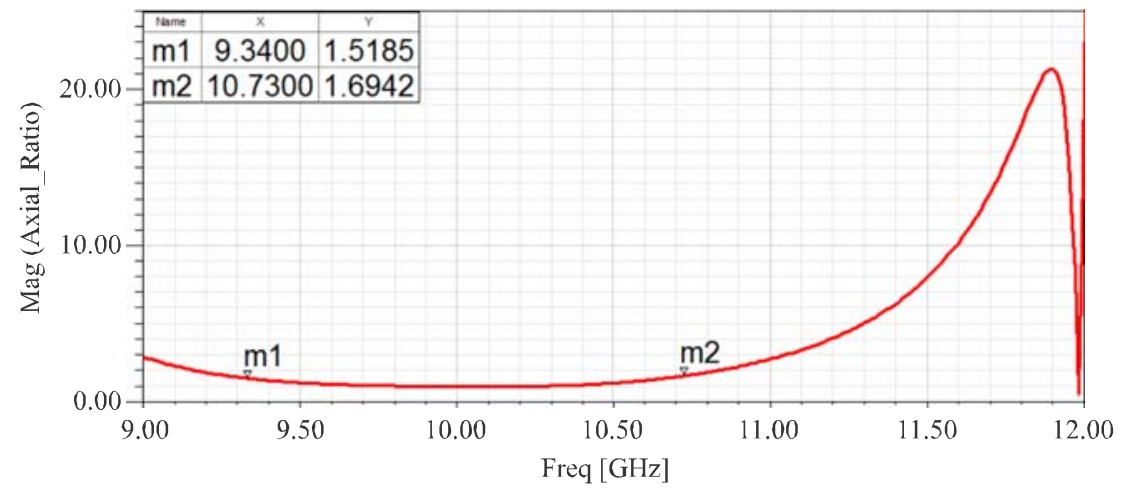

FIG.5. AXIAL RATIO VERSUS FREQUENCY OF SINGLE LAYER POLARIZER

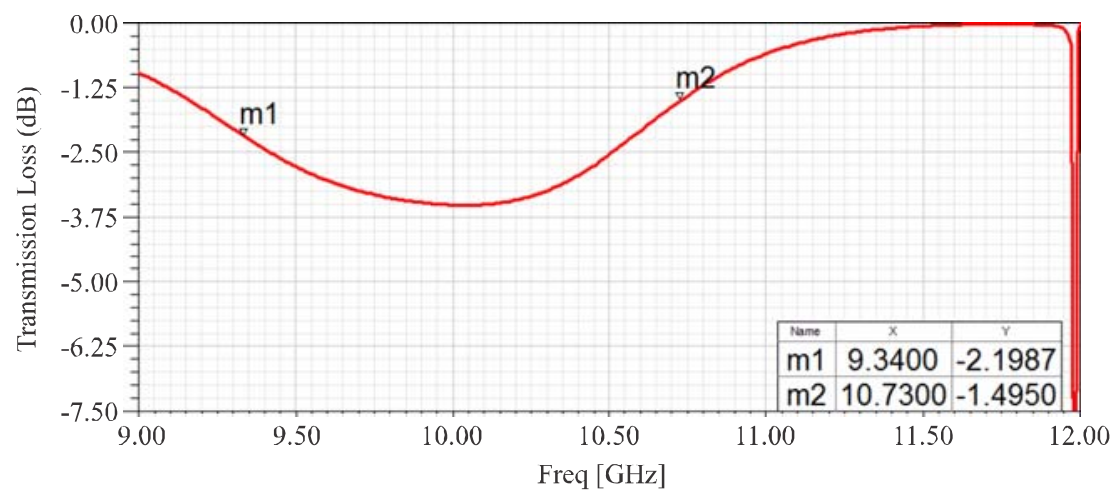

FIG.6. TRANSMISSION LOSS OF E-FIELD VERSUS FREQUENCY OF SINGLE LAYER POLARIZER IS OBTAINED UNDER THE NORMAL INCIDENCE OF X-LINEARLY POLARIZED WAVE

Mehran University Research Journal of Engineering \& Technology, Volume 36, No. 3, July, 2017 [p-ISSN: 0254-7821, e-ISSN: 2413-7219] 
Fig. 8 shows the bandwidth axial ratio obtained ranging from $9.16-10.85 \mathrm{GHZ}=16.89 \%$ bandwidth and $3.1 \mathrm{~dB}$ is axial ratio is calculated between $9.16 \mathrm{and} 10.85 \mathrm{GHz}$, respectively.

Fig. 9 represents the transmittance axial ratio versus frequency 1.4 at $9.58 \mathrm{GHz}$. The fractional bandwidth is enhanced from $9.31-11.31 \mathrm{GHz}=19.49 \%$, respectively.
As shown in Fig. 10, the transmission loss is represented to corresponding resonance frequencies $-22 \mathrm{~dB}$ at 9.59 $\mathrm{GHz}$, respectively.

Fig. 11 represents the phase difference of dual layer circular polarizer. When the polarizer is illuminated by the incidence of polarized wave, the generated two linearly polarized waves from the other side of structure realize

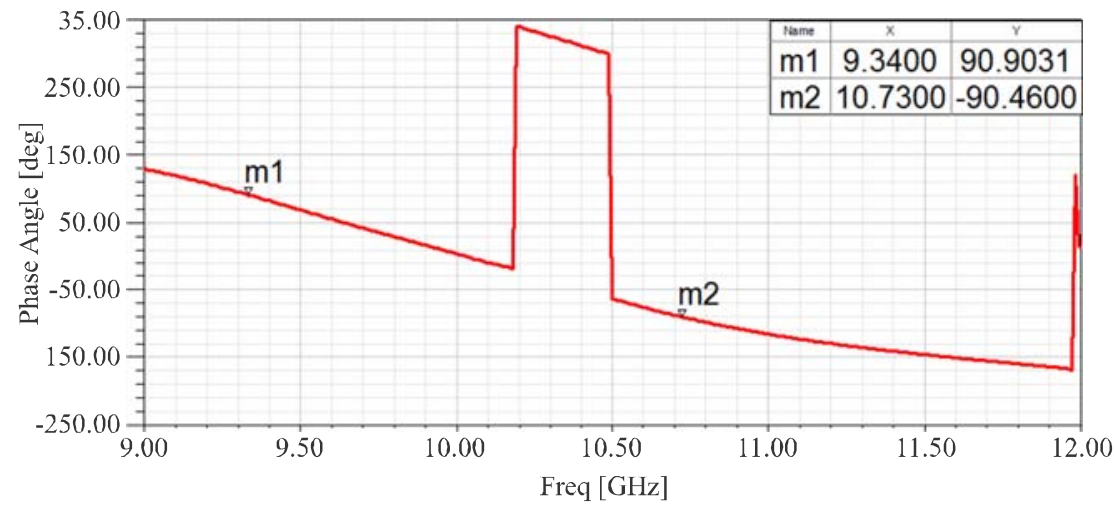

FIG.7. PHASE DIFFERENCE OF E-FIELD VERSUS FREQUENCY OF SINGLE POLARIZER

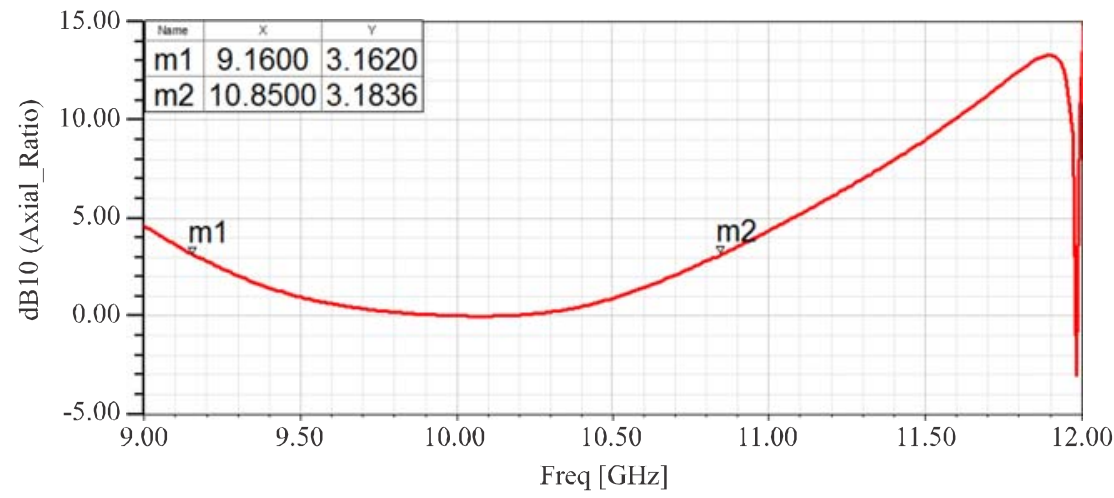

FIG. 8.THE AXIAL RATIO BANDWIDTH VERSUS FREQUENCY OF SINGLE LAYER POLARIZER

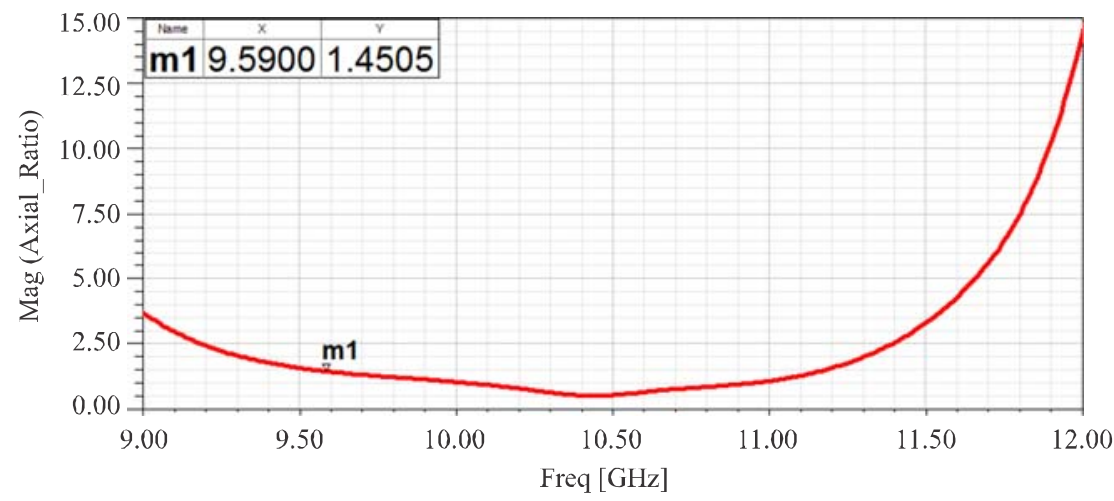

FIG. 9. AXIAL RATIO VERSUS FREQUENCY OF DUAL LAYER POLARIZER

Mehran University Research Journal of Engineering \& Technology, Volume 36, No. 3, July, 2017 [p-ISSN: 0254-7821, e-ISSN: 2413-7219] 
the circular polarized waves along the particular propagation direction. The decomposed orthogonal components $\mathrm{E}_{\mathrm{x}}$ and $\mathrm{E}_{\mathrm{y}}$ have equivalent amplitudes corresponding to $+90^{\circ}$ phase difference at the resonance frequencies. The calculated phase difference of proposed dual layer polarizer is achieved $90.3^{\circ}$ at $10.73 \mathrm{GHz}$, respectively.
Fig. 12 shows the computed axial ratio $3.1 \mathrm{~dB}$ between 9.30-11.31GHz=19.49\% bandwidth and 13.99-14.08GHz= $0.64 \%$, respectively.

The simulation of single and dual-layer circular polarizers are presented which produce the $90^{\circ}$ polarization rotator using single and dual periodic strips along xoy planes to

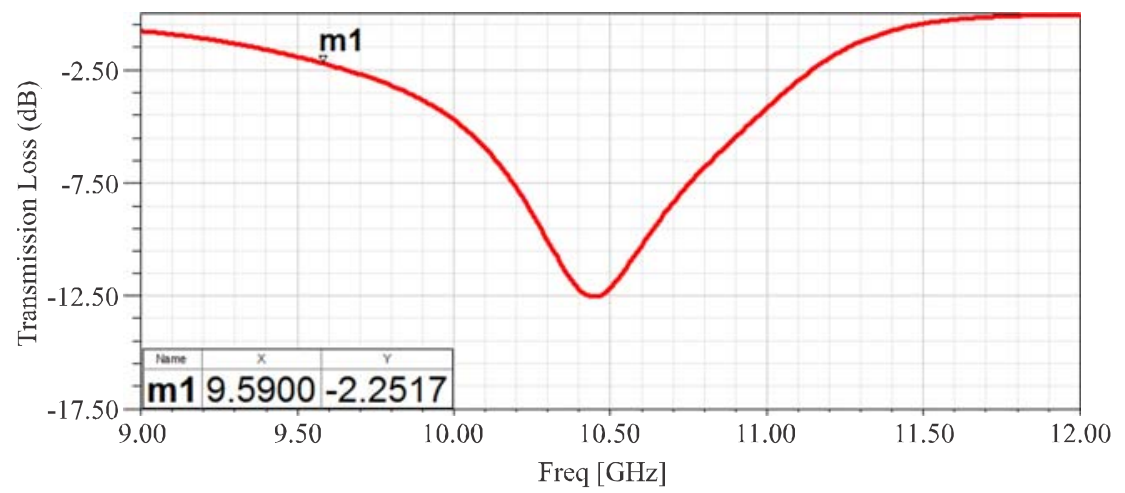

FIG. 10. TRANSMISSION LOSS OF E-FIELD VERSUS FREQUENCY OF DUAL LAYER POLARIZER

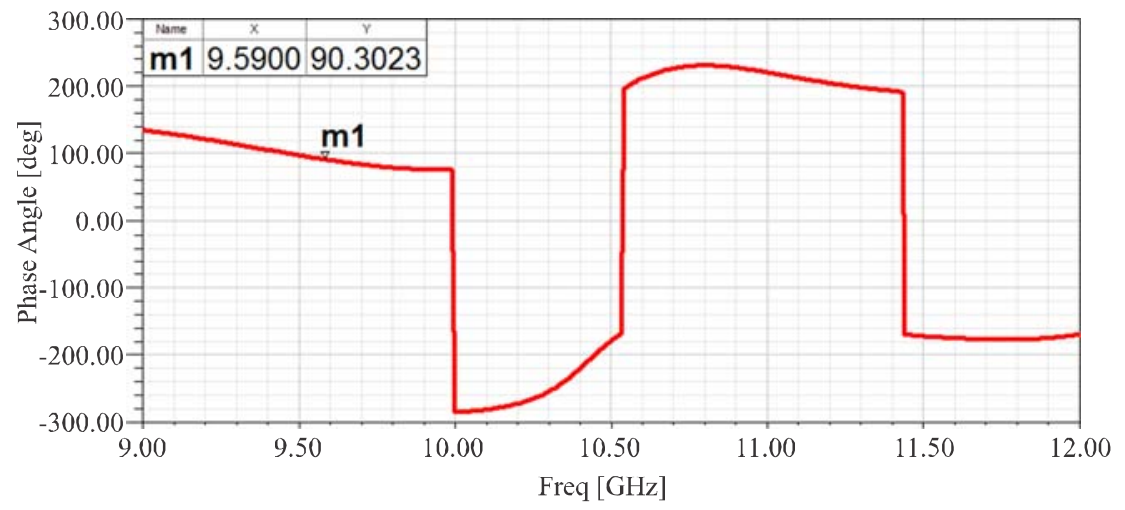

FIG. 11. PHASE DIFFERENCE OF E-FIELD VERSUS FREQUENCY OF DUAL LAYER POLARIZER

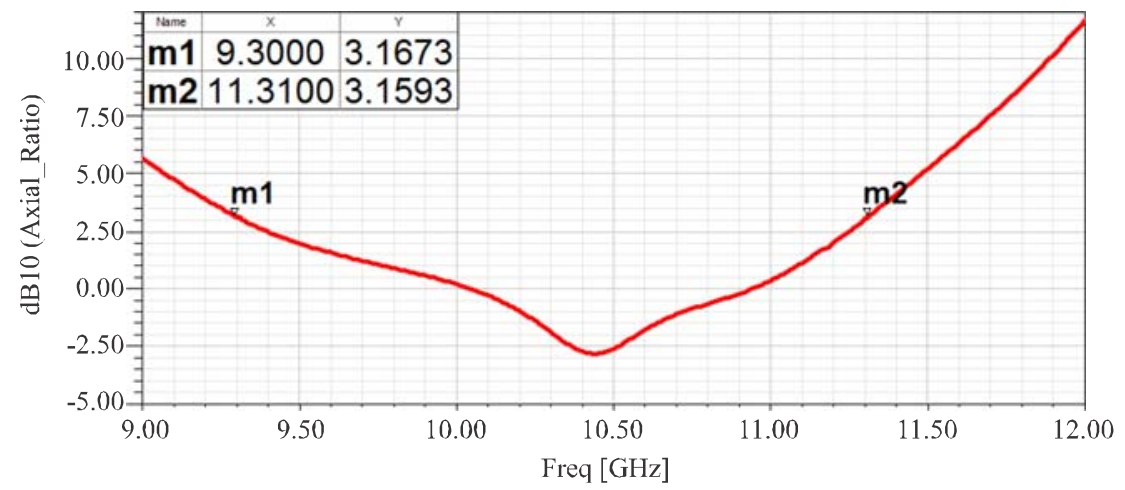

FIG. 12. THE AXIAL RATIO BANDWIDTH VERSUS FREQUENCY OF DUAL LAYER POLARIZER

Mehran University Research Journal of Engineering \& Technology, Volume 36, No. 3, July, 2017 [p-ISSN: 0254-7821, e-ISSN: 2413-7219] 
convert linearly polarized wave into circular polarized wave. The parametric analysis and transmission characteristics of proposed single and dual-layer circular polarized are comprehensively assessed. The good advantages of proposed structures are to achieve optimal bandwidth, low loss transmission, generation of RHCP wave and LHCP wave, good circular polarization corresponding fractional axial ratios over the frequency bands.

Fig. 8 represents the axial ratio bandwidth 9.16$10.85 \mathrm{GHz}=16.89 \%$ of single layer circular polarizer attributes to low loss transmission. We have employed significant technique to enhance the axial ratio bandwidth by using two periodic metallic strips printed on both sides of two substrates. It is observed that the proposed duallayer circular polarizer is achieved transmission with RHCP wave at $9.59 \mathrm{GHz}$ corresponding axial ratio bandwidth ranging from $9.30-11.31 \mathrm{GHz}=19.49 \%$, as shown in Fig. 12 respectively.
The significant advantage of proposed structure is to cover the $x$-band applications and achieve the large bandwidth comparing the various reported designs. In addition, the proposed two layer structure has simple geometry and easy fabrication compared to other designs. Moreover, it can be used as broadband linear polarization converter or as polarization control devices. To better understand the physical behaviour of dual-layer circular polarizer on the transmission peaks, the explanation of the transmission characteristics is provided based on the distribution of the surface current as shown in Fig. 13.

The simulated surface currents at distinct resonance frequency are shown in Fig. 13. It can be clearly observed that the electric charges are largely confined to both metallic strips printed on both sides of the individual substrates. The results show that incident electromagnetic waves excite the resonance at operated frequency. From Fig. 13, it is shown that the surface currents direction on the top and bottom strips is parallel at distinct resonant frequency, which shows the good symmetric transmission originates from the resonance.

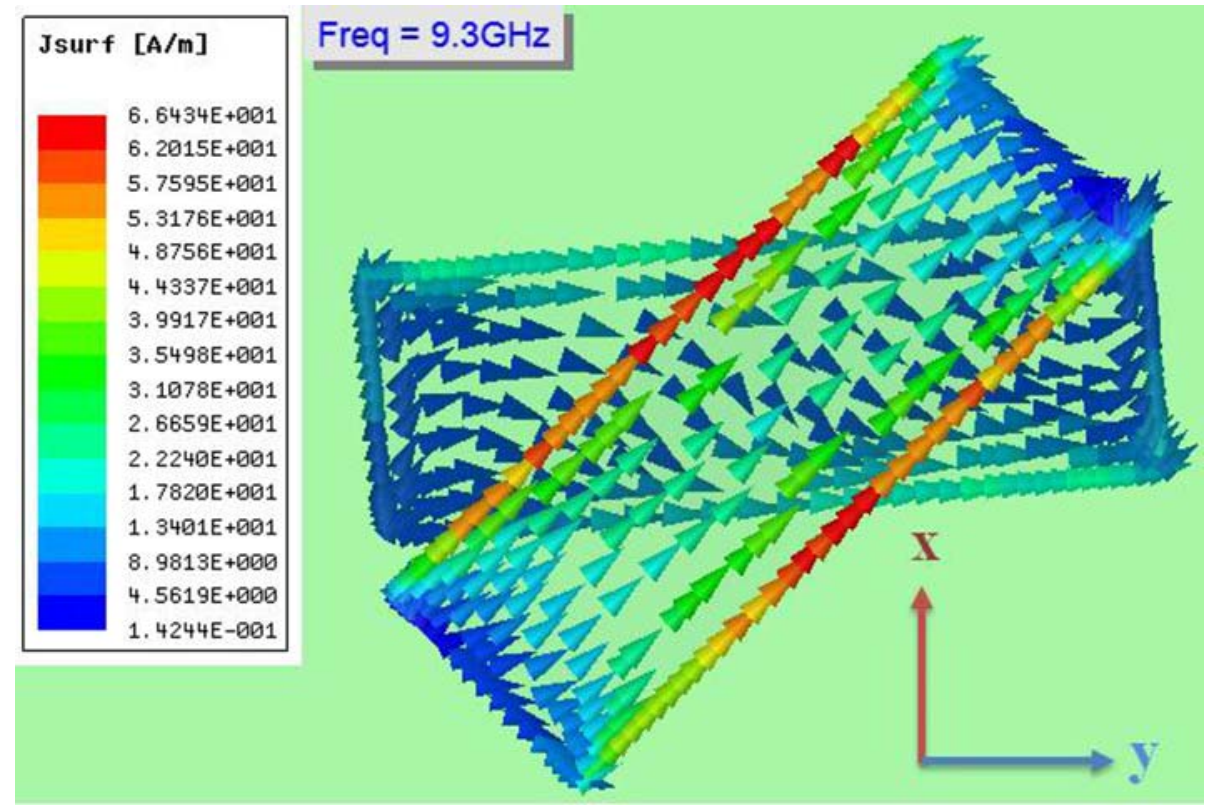

FIG. 13. THE TRANSMISSION ORGINATES FROM RESOANCE FROM DOUBLE-LAYER CIRCULAR POLARIZER

Mehran University Research Journal of Engineering \& Technology, Volume 36, No. 3, July, 2017 [p-ISSN: 0254-7821, e-ISSN: 2413-7219] 


\section{CONCLUSIONS}

In summary, the proposed dual-layer circular polarizer is comprehensively characterized. The various designed structures demonstrated the pure circular polarization with different configurations as reported in this work. The proposed circular polarizer has good circular polarization efficiency with broad operating bandwidth axial ratio which makes potentially useful for $\mathrm{x}$-band applications. The proposed structure has good advantages comparing to reported work, such as, the designed model of single and dual-layer circular polarizer feature exceptionally strong circular polarization with low loss transmission. The HFSS simulation software is used to evaluate the optimal performance of structures. Incident x-linearly polarized wave investigated to clarify the mechanism of polarization transformation. Our designed models are very simple and can be easily fabricated.

The proposed structure has simple geometry but more functionality in distinct frequency bands than previous designs and can be used in various applications, such as, polarization spectral filter, remote sensors, antenna radome and radiometer. The transmission loss and bandwidth widening of structures can be efficiently improved by varying geometric limitations, such as, adding multi-layer, width and length size of strips, substrate thickness, distance adjustment of layers, and printing of strips on the substrate at different direction along xoy planes, respectively.

\section{ACKNOWLEDGEMENT}

First author (Farman Ali Mangi) proposed this idea and constructed methods for this research. Author worked on the designed simulation models and collected the related literature review.

\section{REFERENCES}

[1] Marc-Andre, J., Mathieu, R., and Yves, D., "A MeanderLine Circular Polarizer Optimized for Oblique Incidence”, IEEE Transactions on Antennas and Propagation, Volume 63, No. 12, pp. 5391-5398, Canada, December 2015.

[2] Euler, M., Fusco, V., Cahill, R., and Dickie, R., "Comparison of Frequency Selective Screen-Based Linear to Circular Split-Ring Polarization Convertors”, IET Microwaves, Antennas and Propagation, Volume 4, No. 11, pp. 1764-1772, North Ireland, November, 2010.

[3] Joyal, M., and Laurin, J., “Analysis and Design of Thin Circular Polarizers Based on Meander Lines”, IEEE Transactions on Antennas Propagation, Volume 60, No. 6, pp. 3007-3011, Canada, June, 2012.

[4] Kaschke, J., Gansel, J.K., and Wegener, M., "On Metamaterial Circular Polarizers Based on Metal NHelices”, Optics Express, Volume 20, No. 23, pp. 2601226020, Germany, November, 2012.

[5] Li, Y.F., Zhang, J.Q., Qu, S.B., Wang, J.F., Chen, H.Y., $\mathrm{Xu}, \mathrm{Z}$., and Zhang, A.X., "Wideband Selective Polarization Conversion Mediated by ThreeDimensional Metamaterials”, Journal of Applied Physics, Volume 115, No. 123, pp. 234506-1-234506-6, Chine, June, 2014.

[6] Huang, X.J., Yang, D., and Yang, H.L., "Multiple-Band Reflective Polarization Converter Using U-Shaped Metamaterial”, Journal of Applied Physics, Volume 115, No. 10, 103505-1-103505-6, China, March, 2014.

Xu, H.X., Wang, G.M., and Qi, M.Q., “Compact DualBand Circular Polarizer Using Twisted Hilbert-Shaped Chiral Metamaterial”, Optic Express, Volume 21, No. 21, pp. 24912-24921, China, October, 2013.

[8] Yan, S., and Vandenbosch, G.A.E., "Compact Circular Polarizer Based on Chiral Twist Double Split-Ring Resonator”, Applied Physics Letters, Volume 102, No. 10, pp. 103503-103503-4, Belgium, March, 2013.

Mehran University Research Journal of Engineering \& Technology, Volume 36, No. 3, July, 2017 [p-ISSN: 0254-7821, e-ISSN: 2413-7219] 
[9] Zhu, H.L., Cheung, S.W., Chung, K.L., and Yuk, T.I., "Linear-to-Circular Polarization Conversion Using Metasurface,” IEEE TransactionsAntennas Propagation, Volume 61, No. 9, pp. 4615-4623, China, September, 2013.

[10] Mutlu, M., Akosman, A.E., and Ozbay, E., "Broadband Circular Polarizer Based on High-Contrast Gratings”, Optic Letters, Volume 37, No. 11, pp. 2094-2096, Turkey, June, 2012.

[11] Mutlu, M., Akosman, A.E., Kurt, G., Gokkavas, M., and Ozbay, E., "Experimental Realization of a High-Contrast Grating Based Broadband Quarter-Wave Plate”, Optics Express, Volume 20, No. 25, pp. 27966-27973, Turkey, December, 2012.

Strikewerda, A., "Comparison of Birefringent Metamaterials and MeanderlineStructure as QuarterWave Plates at Terahertz Frequencies”, Optics Express, Volume 17, No. 7, pp. 136-149, USA, January, 2009.
[16] Karkkainen, K., and Stuchly, M., "Frequency Selective Surface as a Polarization Transformer”, IEE Proceedings on Microwaves, Antennas and Propagation, Volume 149, Nos. 5-6, pp. 248-252,Finland, October-December, 2002.

[17] Masa-Campos, J.L., and Gonz'alez-Fern’andez, F., "Dual Linear/Circular Polarized PlannarAntenna with Low Profile Double-Layer Polarizer of $45^{\circ}$ Tilted Metallic Strips for Wimax Applications”, Progress in Electromagnetics Research, PIER, Volume 98, pp. 221-231, Spain, 2009.

[18] Sohail, I., Ranga, Y., and Karu, P.E., “A Linear to Circular Polarization Converter Based on Jerusalem-Cross Frequency Selective Surface”, 7th European Conference on Antennas and Propagation, pp. 2141-2143, April, 2013.

[19] Mangi, F.A., Xiaoand, S., and Mallah, G.A., "Multiband Circular Polarizer Based on Fission Transmission of Linearly Polarized Wave for X-Band Applications”, Journal of Electrical \& Computer Engineering, pp. 1-8, China, July, 2016.

[20] Mangi, F.A., Mallah, G.A., and Shah, A.A., "Novel Broadband FSS Dual-Layer Circular Polarizer Based on Quarter Wave Plate”, Volume 48, No. 1, pp. 125-130, China, 2016.

[21] Zhou,Z and Yang, H,"Triple-Band Asymmetric Transmission of Linear Polarization with Deformed SShape Bilayer chiral metamaterial”, Applied Physics, Volume 119, No. 1, pp. 115-119,USA, 2015.

[22] He-Xiu X., Guang-Ming, W.M., and Qing, Q., "DualBand Circular Polarizer and Asymmetric Spectrum Filter using Ultrathin Compact Chiral Metamaterial”, Progress in Electromagnetics Research, Volume 143, pp. 243-261, China, 2013. 